\title{
The Virus is Coming! The Ways the Canadian-Born and Foreign-Born Changed Weekly Activities During the Pandemic ${ }^{1}$
}

Fernando Mata

School of Sociological and Anthropological Studies

University of Ottawa

fmata@uottawa.ca

April 30, 2021

\begin{abstract}
During epidemics, individuals change their weekly activities to increase their sense of personal security, health, efficacy and comfort. This study looks at changes in weekly activities reported by Canadians and foreign-born citizens during the first wave of the COVID-19 pandemic in Canada. The data for the study is drawn from a combined survey of two cycles of the CPSS (Canadian Perspective Series) surveys 2 and 4 which were conducted between May 4 and July 26, 2020. The combined survey consisted of 8,818 adult respondents $(7,280$ Canadian-born and 1,538 Foreignborn respondents). Weekly activities were measured by 25 survey questions which collected information on habits such as washing hands, avoiding crowds, watching more T.V., internet use and exercising. Principal Component Analysis (PCA) was used as the main tool for the statistical analysis of the data. Statistical testing of mean differences revealed that Canadian and foreign-born groups differed from each other in 15 out of the 25 activity items tested. PCA identified six major domains of weekly activities. Immigrants displayed similar activity patterns compared to the Canadian-born concerning the Sanitary, Leisure, Planning and Hoarding domain of activities but were found somewhat dissimilar in terms of the Sheltering and Substance use activity domains. During the pandemic lockdown, immigrants "hunkered down" and were more proactive in adopting sheltering types of activities such as cancelling travel plans, exercising more indoors than outdoors, practicing meditation and making better food choices. In doing so, they also avoided substance use as pandemic coping activities such as higher consumption of alcohol, tobacco and cannabis compared to the Canadian-born.
\end{abstract}

\subsection{Introduction}

The COVID-19 pandemic has disrupted the lives of people in Canada and worldwide ${ }^{2}$. The first wave of the pandemic (March-September 2020) in Canada was particularly troubling because of the novelty of lockdowns and the imposition of severe sanitary guidelines which created an unusual level of collective anxiety. On March 11, when the World Health Organization declared COVID-19 a pandemic, Canada had already more than 100 virus cases ${ }^{3}$. On March 14, the federal government urged Canadians abroad to return home as soon as possible as borders were closing. By April 22, Ontario and Quebec, the hardest-hit provinces, called on the military to help out in long-term care homes. On June 18, Canada officially recorded more than 100,000 cases of COVID-19. The sequence of all these events alerted the Canadian public to the seriousness of the sanitary situation in the country.

\footnotetext{
${ }^{1}$ Paper to be presented at the CSA Meetings, University of Victoria, B.C, June 4-8, 2022. The author would like to thank Jennifer Domoulin, Stein Monteiro (CERC), the Ontario Data Documentation, Extraction Service and Infrastructure (ODESI-Scholars Portal Statistics) as well as Statistics Canada for making available the data and valuable support and guidance.

${ }^{2}$ Pandemic declared by the World Health Organization (WHO) on 31st January 2020 (WHO, 2020).

${ }^{3}$ Source: https:/ / www.ctvnews.ca/health/coronavirus/grim-anniversary-a-timeline-of-one-year-of-covid-19-1.5280617, retrieved 2021-04-24.
} 
The epidemiological literature suggests that, during epidemics, to minimize health risks, individuals change the nature of their routine activities (Strong, 1990; Hunt, 1999). These changes are said to provide individuals with a greater sense of personal security, health, efficacy and comfort (Lazarus, 1986; Kelly, 2019). Fear is also mentioned as a major driver behind these observed behavioral changes (Maeng \& Milad, 2015; Manderson and Levine, 2020). With social ties disrupted and more time to be spent at home, the period of COVID-19 confinement has led to a soul-searching process where individuals are finding meaning in their lives by performing essential survival tasks.

Immigrant sub-populations in various host countries, which include Canada, have been identified as among high risk COVID 19 groups in society (OECD,2020; IRPP,2020). Immigrants, particularly those of recent stay, have a range of vulnerabilities such as higher incidence of poverty, overcrowded housing conditions and a higher occupational concentration in jobs where physical distancing is difficult. Considering that immigrants may be differentially exposed to COVID-19 risks, it is appropriate to ask if their weekly activities would be substantially different from those of their Canadian-born counterparts. For instance would they be more observant of sanitary precautions such as washing hands, avoiding crowds or distancing from crowds when compared to those reported by the Canadian-born? Would the reporting of weekly activities also be different in the areas of leisure, social inter-connectedness or those practices related to mental or physical health? Aside from immigrant status, what other sociodemographic and residential correlates may have also had an impact on these behavioral responses? Using combined information from two cycles of the Canadian Survey Perspectives Surveys (CPSS) of the adult population of Canada, this study addresses these research questions and reflects on the role that immigrant status played in the types of weekly activities being reported.

\subsection{Data, Measures and Analytical Methods}

The data for this study is drawn from a combined sample of the public files of CPSS 2 and CPSS 4 surveys conducted by Statistics Canada in 2020. These are ongoing surveys regarding public views on the socio-economic impacts of the pandemic on Canadian society. The first CPSS survey was conducted between May 4-10, 2020 and the second one between July 20-26, 2020. These surveys are short online voluntary surveys comprising approximately 4,000 Canadians aged 15 years old and over who are residents of the 10 Canadian provinces. The probability selection panels of CPSS surveys are created by randomly selecting a subset of the larger Labour Force Survey (LFS).

Comparing Canadian and foreign-born survey respondents to the CPSS surveys presents sample size challenges as immigrants regularly only comprise about $20 \%$ of each survey cycle (about 800 individuals or less). Pooling respondents from two CPSS surveys offered important advantages for statistical analysis. First, a larger sample size yields correspondingly larger foreign-born population coverage, thereby increasing the level of confidence in any point estimates and therefore decreasing standard errors of population parameters. Second, a larger immigrant sample allows for a better exploration of weekly activities in sub-groups such as those living in urban areas and those living in more crowded conditions such as those living in apartment buildings where conditions of virus transmission are higher.

The merging of the CPSS surveys produced a dataset comprising of 8,818 individuals $(4,600$ from CPSS 2 and 4,218 from CPSS 4) ${ }^{4}$. This combined survey represented a weighted population of 62.2 million adult Canadians across two cycles ${ }^{5}$. The addition of the foreign-born contingent of CPSS $4(n=746)$ to CPSS 2

\footnotetext{
${ }^{4}$ The merging of the two survey datasets required identifying all common variables of interest ensuring they were standardized to a common coding.

${ }^{5}$ The logic guiding the weighting of the datasets is that each sample of CPSS respondents is drawn from two similar populations (i.e. two identical adult Canadian populations) measured at two points in time, keeping intact the original weight assigned to
} 
$(\mathrm{n}=796)$ raised the total of foreign-born respondents to 1,538 which represents approximately 15.1 million Canadian adults.

In the CPSS surveys, immigrant status was measured by a simple question asking respondents if they were born in Canada or not. In the public files, there is no information on the time spent in Canada or their conditions of admission such as economic, family, or refugee class. Compared to the Canadian-born, the foreign-born population of the pooled survey had a slight overrepresentation of individuals aged 35-44 years old (19\% to 15\%), those reporting married or common-law marital status $(67 \%$ to $60 \%)$, and those who were living with a child under 18 at home $(40 \%$ to $31 \%)$. About $40 \%$ of immigrants had a university education equivalent to a bachelor's degree or above from a university, compared to only $25 \%$ among the Canadian-born. Also, $44 \%$ lived in high-rise apartments or other accommodations arrangements compared to only $20 \%$ among the Canadian-born who mostly lived in detached houses (69\%).

Weekly activity items were collected from a set of 25 survey questions where respondents were asked about frequencies of typical weekly activities undertaken during the reference week of the surveys ${ }^{6}$. Activity items were measured as dichotomous scales $(0=$ if activity was not reported, $1=y e s$ if the activity was reported). These activities comprised the following: washing hands, physically distancing from others, avoiding crowds, not leaving the house, not touching the face, working more from home, communicating with friends \& family, making care plans for household and non-household members, developing communication plans, canceling travel, exercising outdoors, exercising indoors, practicing meditation, filling in prescriptions, stocking up on essentials and making better food choices. Activity items also included reported increases in activities such as internet use, T.V. watching, junk food consumption, playing videogames, board games playing as well as alcohol, tobacco and cannabis consumption.

PCA (Principal Components Analysis) was the main multivariate tool used in the analysis. PCA is a factor analytic technique that builds a sequence of uncorrelated (orthogonal) linear combinations called components (Everitt and Dunn, 2001). PCA reduces the multidimensionality of the data to a simpler structure to account for the largest possible portions of data variation. PCA's perceptual and/or behavioural maps (also called "correlation circles") are also useful here as they visualize information allowing for a focus on individual groups and their coordinate positions in a multidimensional plane (Greenacre, 2010). It should be noted that PCA is used in this paper for exploratory purposes only and not confirmatory ones. It is used to capture as many latent variables $^{7}$ (domains of activities) as possible underlying the spectrum of weekly activities reported by survey respondents.

individual respondents representing these two populations. This strategy is better than just dividing the original weight by 2 (i..e the number of cycles) .

${ }^{6}$ The COVID-19 precautions (Questions BH20A to BH20_N) were phrased : Which of the following precautions have you taken to reduce your risk of exposure to COVID-19? (List provided). The health activity (Questions BH35A to BH35_E) were phrased : Are you doing any of the following activities for your health? (List provided). The weekly habits (Questions BH40A to BH40_H) were phrased: Have your weekly habits changed for any of the following activities? (List provided) .

In psychometrics, a latent variable is a hypothetical construct explain an observed covariation in behaviour. 


\subsection{Findings}

Table 1 presents the weekly activity items (\% reported) for Canadian and Foreign-born respondents to the CPSS surveys. Overall, the most mentioned weekly activities by members of both sub-populations were those related to regularly washing hands (94\%) followed by physical distancing in public (91\%), plans to communicate with others (91\%), and avoiding crowds or large gatherings (90\%).

In total, 15 out of the 25 group means difference tests contrasting Canadian and foreign-born respondents to the survey were found statistically significant at the $p<.05$ level. In terms of the most significant differences found, the foreign-born were the most likely to quickly cancel travel ( $+11 \%$ points difference), exercise indoors $(+12 \%$ points difference) and practice meditation $(+11 \%$ points difference) compared to the Canadian-born. Also noticeable to a minor extent is a reported increase among the foreign-born in making better food choices and internet use time ( $+7 \%$ points difference respectively). In contrast to the Canadian-born, immigrants were less likely to report exercising outdoors (-12\% points difference) and/or report an increase in alcohol consumption (-10\% points difference). Differences ranging from $+5 \%$ and $5 \%$ were found for the remaining items.

Table 1: Weekly Activity (\% reported) by Immigrant Status and Statistical tests results, Canadian-born vs. Foreignborn respondents to the combined CPSS survey, Canada 2020

\begin{tabular}{|c|c|c|c|c|c|c|}
\hline Item Number & Weekly Activities & All & Foreign & Canadian & Diff (FB & t-test, sig. \\
\hline 1 & Washed hands & $94 \%$ & $94 \%$ & $94 \%$ & $0 \%$ & no \\
\hline 2 & Physically distanced from others & $91 \%$ & $91 \%$ & $91 \%$ & $0 \%$ & no \\
\hline 3 & Made communication plans & $91 \%$ & $90 \%$ & $91 \%$ & $-1 \%$ & no \\
\hline 4 & Avoided crowds & $91 \%$ & $92 \%$ & $90 \%$ & $+2 \%$ & no \\
\hline 5 & Not left the house & $82 \%$ & $83 \%$ & $80 \%$ & $+3 \%$ & yes \\
\hline 6 & Exercised outdoors & $69 \%$ & $63 \%$ & $75 \%$ & $-12 \%$ & yes \\
\hline 7 & Not touching face & $71 \%$ & $72 \%$ & $69 \%$ & $+4 \%$ & yes \\
\hline 8 & Increase in internet time & $65 \%$ & $68 \%$ & $61 \%$ & $+7 \%$ & yes \\
\hline 9 & Stocked up on essentials & $56 \%$ & $58 \%$ & $53 \%$ & $+5 \%$ & yes \\
\hline 10 & Increased in T.V. watching & $54 \%$ & $56 \%$ & $52 \%$ & $+4 \%$ & no \\
\hline 11 & Exercised indoors & $57 \%$ & $63 \%$ & $51 \%$ & $+12 \%$ & yes \\
\hline 12 & Cancelled travel & $48 \%$ & $53 \%$ & $42 \%$ & $+11 \%$ & yes \\
\hline 13 & Communicated with friends \& family & $40 \%$ & $38 \%$ & $41 \%$ & $-3 \%$ & no \\
\hline 14 & Making better food choices & $44 \%$ & $47 \%$ & $40 \%$ & $+7 \%$ & yes \\
\hline 15 & Filled prescriptions & $30 \%$ & $26 \%$ & $33 \%$ & $-7 \%$ & yes \\
\hline 16 & Increased junk food consumption & $30 \%$ & $28 \%$ & $32 \%$ & $-4 \%$ & yes \\
\hline 17 & Worked more from home & $29 \%$ & $30 \%$ & $28 \%$ & $+2 \%$ & no \\
\hline 18 & Increase in playing videogames & $22 \%$ & $21 \%$ & $23 \%$ & $-2 \%$ & no \\
\hline 19 & Practiced meditation & $27 \%$ & $32 \%$ & $21 \%$ & $+11 \%$ & yes \\
\hline 20 & Increase in board game playing & $19 \%$ & $16 \%$ & $21 \%$ & $-5 \%$ & yes \\
\hline 21 & Increase in alcohol consumption & $15 \%$ & $10 \%$ & $20 \%$ & $-10 \%$ & yes \\
\hline 22 & Made care plans for non-household members & $14 \%$ & $12 \%$ & $16 \%$ & $-4 \%$ & yes \\
\hline 23 & Made care plans for household members & $13 \%$ & $13 \%$ & $12 \%$ & $+1 \%$ & no \\
\hline 24 & Increase in cannabis consumption & $6 \%$ & $4 \%$ & $7 \%$ & $-3 \%$ & yes \\
\hline 25 & Increase in tobacco consumption & $5 \%$ & $4 \%$ & $5 \%$ & $-1 \%$ & no \\
\hline
\end{tabular}

Source: Combined Survey, CPSS Series 2 \& 4 Surveys 2020, Statistics Canada

The highest inter-item correlations found were those of internet use and T.V watching $(\mathrm{r}=+.46)$, physical distancing and avoiding crowds $(\mathrm{r}=+.37)$, stocking up on essentials and filling prescriptions $(\mathrm{r}=+.35)$ and not touching face and washing hands $(\mathrm{r}=+.34)$. To identify the underlying domains of the weekly activity items, principal component analysis (PCA) was applied to the inter-correlation matrix of activity items. In 
total, eight components with eigenvalues greater than one ${ }^{8}$ were extracted but only six were kept as the last two ones loaded on one variable only each and contributed very little to the total variance explained.

The six major components extracted from the item inter-correlation matrix accounted for $41.2 \%$ of the total variance of the 27 items (see table 2 ). The first component (12.2\% of variance explained) highly loaded on sanitary measures followed such as avoiding crowds, hand-washing, physical distancing, not touching face, and/or not leaving the house. The second component (accounting for $8.0 \%$ of the total variance) did so with respect to leisure activities such as internet use, T.V watching, and others. These two domains were labeled Sanitary and Leisure, respectively. The third component $(6 \%$ of the variance explained) named Communications tapped activities related to caring for household and non-household members and general communication plan. The fourth component ( $5.7 \%$ of explained variance) measured a special combination of activities such as exercising indoors, doing meditation and/or developing better food choices (loadings of $.67, .65$ and .64 respectively). It also had a moderate loading on the cancellation of travel (loading=.31). This latter domain, labeled Sheltering ${ }^{9}$, is where immigrants clearly differentiated themselves from the Canadian-born according to the previous t-tests carried out. The fifth component (5.0\% of variance explained) tapped into a Hoarding type of domain were stocking up on essentials and filling prescriptions are the main activities carried out while the last component (4.3\% of variance explained), labeled Substance Use domain, tapped into greater consumption of cannabis, tobacco and alcohol.

The behavioural map of weekly activity items in the major domains of activity items is presented in chart $1^{10}$. Combined, these two domains account for one-fifth $(20.2 \%)$ of the total variation of activity items. The vector positions of the Canadian-born and foreign-born groups appear opposite to each other as these are mutually exclusive categories. Both groups appear equally distant from the vectors representing sanitary activities such as washing hands, keeping distances or avoiding crowds suggesting no major differences between the two groups in terms of the performing of weekly activities. However, the foreign-born group's position in the component plane is closer to Sheltering type of activities such as cancelling travel, indoor exercising, practicing meditation and developing better food choices while they are more distant concerning the substance use items which are relatively closer to the Canadian group one. Acute angles between Sheltering type activity items reveal strong correlations between these four activity items.

A component (sometimes called factor) score is a numerical value that indicates a person's relative spacing or standing on an extracted component. Component scores are measured on a standardized scale (mean=0, standard deviation= 1 ). An average positive component score represents higher scores on the major constituent variables of the components and a negative one the opposite. Average component scores in the six major domains for the Canadian-born and foreign-born groups are presented in Chart 2 . Results of independent sample t-tests conducted on these component scores suggests that the most significant and substantive differences are found concerning the Sheltering and Substance use domains ${ }^{11}$. Average scores for the first domain of interest are .26 standard deviations below the zero mean while

\footnotetext{
${ }^{8}$ Kayser-Guttman criteria for determining factor or component importance. Loadings $>$ (abs).33 are regarded here as acceptable items in the configuration of component domains.

${ }^{9}$ Sheltering is interpreted here as "hunkering-down" or settling into the safety of one's home or shelter for a potentially prolonged time. This type of behaviour has been observed more frequently during natural disasters or the outbreak of a contagious disease.

${ }^{10}$ In behavioural maps the positions of groups of interest vs. their reported behaviours is effectively visualized. In the plot, vectors represent items and groups which are presented as points in space. Correlations between two vectors in component space are equal to the cosines of the angles between the indicator vectors $(\theta)$, or $r=\cos (\theta)$. Highly correlated variables are located at sharp angles from each other ( $\theta=90$ degrees or less) while those zero correlated are "orthogonal" to each other $(\theta=90$ degrees). If variables are perfectly negatively correlated, then they are placed at obtuse angles (vector in opposite direction).

${ }^{11}$ In an oblique rotation procedure of the item correlation matrix which allowed for factor or component inter-correlations, the Sheltering component was found negatively correlated with the Substance Use one $(r=-.05)$.
} 
those of the Canadian-born -.09 standard deviations below it. In second domain, foreign-born are located -.15 standard deviations below the zero mean while the Canadian-born .04 standard deviations above it.

Table 2: Varimax Rotated Component Loadings (ranked by size*) of First Six Extracted Components, Weekly Activity Items, Canada 2020

\begin{tabular}{|c|c|c|c|c|c|c|c|}
\hline $\begin{array}{l}\text { Item } \\
\text { Number }\end{array}$ & Weekly Activity Items & $\begin{array}{l}\text { C1: } \\
\text { Sanitary } \\
\text { Domain }\end{array}$ & $\begin{array}{l}\text { C2: } \\
\text { Leisure } \\
\text { Domain }\end{array}$ & $\begin{array}{l}\text { C3: } \\
\text { Planning } \\
\text { Domain }\end{array}$ & $\begin{array}{l}\text { C4: } \\
\text { Sheltering } \\
\text { Domain }\end{array}$ & $\begin{array}{l}\text { C5: } \\
\text { Hoarding } \\
\text { Domain }\end{array}$ & $\begin{array}{l}\text { C6: } \\
\text { Substance Use } \\
\text { Domain }\end{array}$ \\
\hline 1 & Avoiding crowds & 0.71 & -0.01 & 0.04 & 0.00 & 0.09 & -0.01 \\
\hline 2 & Washing hands & 0.68 & 0.06 & 0.04 & -0.05 & 0.02 & -0.01 \\
\hline 3 & Physically distancing & 0.65 & 0.03 & 0.01 & 0.04 & 0.10 & 0.00 \\
\hline 4 & Not touching face & 0.59 & 0.03 & 0.17 & 0.04 & -0.04 & -0.04 \\
\hline 5 & Not leaving house & 0.44 & 0.07 & 0.02 & 0.08 & 0.36 & 0.01 \\
\hline 6 & Internet time increase & 0.04 & 0.75 & 0.03 & 0.10 & 0.11 & 0.00 \\
\hline 7 & T.V. watching increase & 0.04 & 0.75 & -0.03 & 0.05 & 0.14 & 0.01 \\
\hline 8 & Videogames increase & 0.05 & 0.58 & -0.01 & 0.05 & -0.13 & 0.15 \\
\hline 9 & Junk food increase & -0.02 & 0.47 & 0.07 & -0.17 & 0.07 & 0.26 \\
\hline 10 & Boardgames increase & 0.05 & 0.40 & 0.20 & 0.05 & -0.19 & -0.11 \\
\hline 11 & Non-household care plans & 0.05 & 0.02 & 0.75 & 0.02 & 0.05 & 0.01 \\
\hline 12 & Household care plans & 0.02 & 0.01 & 0.74 & 0.06 & 0.11 & 0.06 \\
\hline 13 & Communication plans & 0.19 & 0.09 & 0.45 & 0.12 & 0.28 & -0.03 \\
\hline 14 & Cancelling travel & 0.30 & 0.07 & 0.06 & 0.31 & -0.09 & -0.08 \\
\hline 15 & Indoor exercise & 0.08 & 0.06 & -0.01 & 0.67 & -0.03 & -0.08 \\
\hline 16 & Making better food choices & 0.05 & 0.12 & 0.06 & 0.65 & -0.01 & 0.06 \\
\hline 17 & Meditation & -0.10 & -0.08 & 0.12 & 0.64 & 0.05 & 0.06 \\
\hline 18 & Stocked up on essentials & 0.08 & 0.09 & 0.11 & 0.07 & 0.73 & -0.06 \\
\hline 19 & Filled prescriptions & 0.06 & -0.03 & 0.15 & -0.10 & 0.73 & 0.03 \\
\hline 20 & Cannabis Increase & 0.02 & 0.07 & -0.02 & 0.09 & -0.02 & 0.74 \\
\hline 21 & Tobacco Increase & -0.06 & 0.02 & 0.00 & 0.01 & 0.00 & 0.72 \\
\hline 22 & Alcohol increase & -0.07 & 0.20 & 0.11 & -0.19 & -0.07 & 0.43 \\
\hline 23 & Outdoors exercise & 0.06 & -0.09 & 0.01 & 0.07 & 0.00 & -0.03 \\
\hline 24 & Communication plans & 0.16 & 0.10 & 0.00 & 0.16 & 0.13 & 0.08 \\
\hline \multirow[t]{4}{*}{25} & Working from home & 0.08 & -0.01 & 0.06 & 0.09 & 0.04 & -0.03 \\
\hline & Eigenvalue & 3.04 & 2.00 & 1.50 & 1.42 & 1.26 & 1.08 \\
\hline & $\%$ of Explained Variance & 12.2 & 8.0 & 6.0 & 5.7 & 5.0 & 4.3 \\
\hline & Cumulative \% Variance & 12.2 & 20.2 & 26.3 & 31.9 & 36.9 & 41.2 \\
\hline
\end{tabular}

* Shaded=loadings with an absolute value of .30 or higher. Source: Combined survey, CPSS Series $2 \& 4$ cycles 2020, Statistics Canada 
Chart 1: PCA's Behavioural Map of Weekly Activity Items, Sanitary and Leisure Weekly Activity Domains, Canadian-born and Foreign-born, Canada 2020

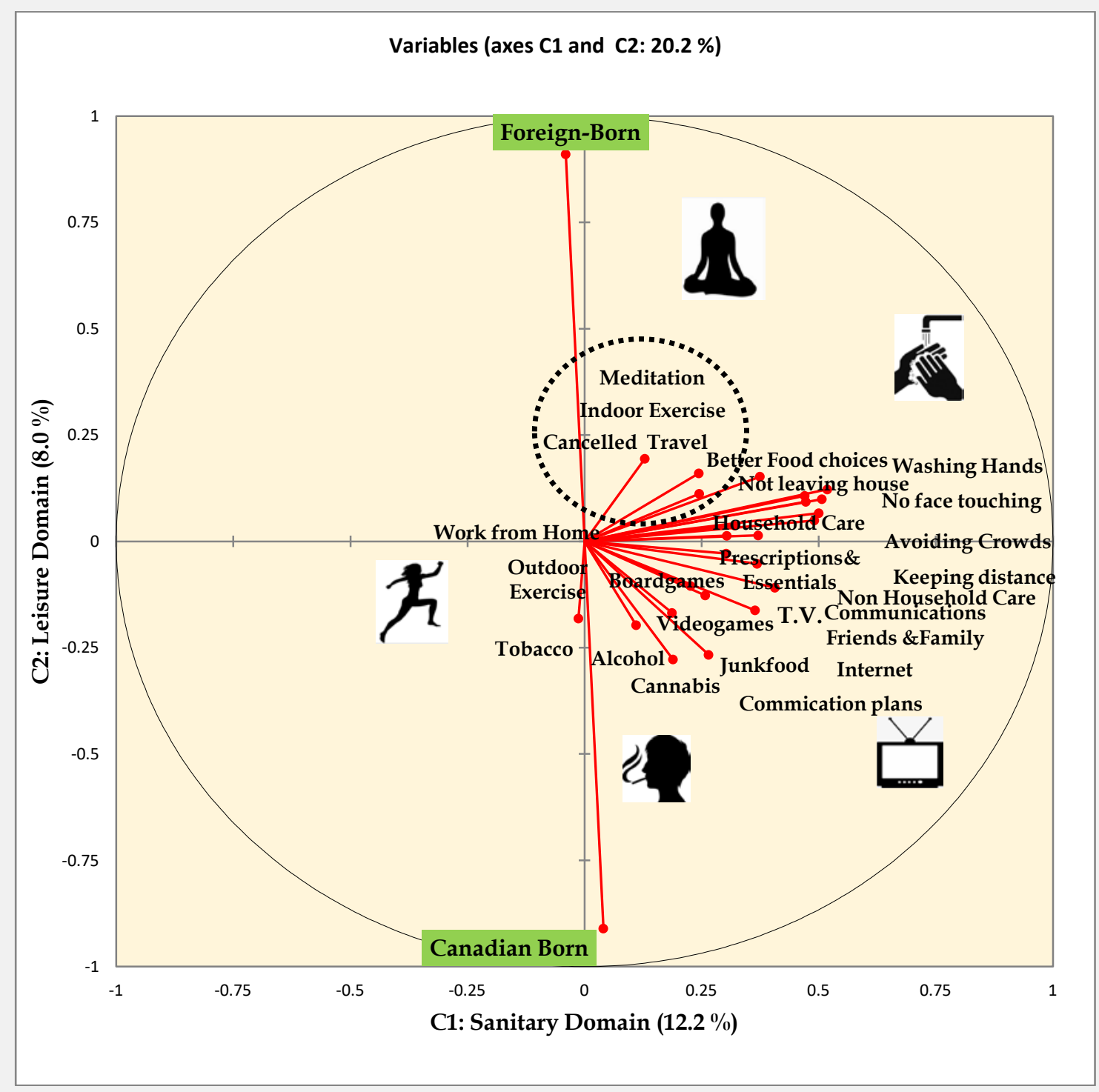

Source: Combined Survey, CPSS Series $2 \& 4$ cycles 2020, Statistics Canada

Having found the general pattern of differences between subpopulations and using the condensing or data reduction properties of factor scores, it is relevant to break down the Canadian-born and foreignborn populations to pinpoint groups where these differences are maximized or minimized. Chart 4 presents selected average component scores for selected socio-demographic and residential groupings. Those foreign-born, living in low-rise apartments with less than 5 floors displayed the highest average positive scores in the Sheltering domain followed by those living with children and/or who had lower levels of education. Overall, most of all immigrant groupings followed the pattern of higher average Sheltering domain scores accompanied by lower Substance Use scores. Patterns for the Canadian-born groupings are mixed. Higher Substance Use scores were found for the younger cohorts of Canadians 
(Under 35 years old and 35-44 years old) as well as for those living in low-rise apartments with less than 5 floors.

Chart 2: Average Component Scores of Six Major Domains of Weekly Activity Items*, Canada 2020

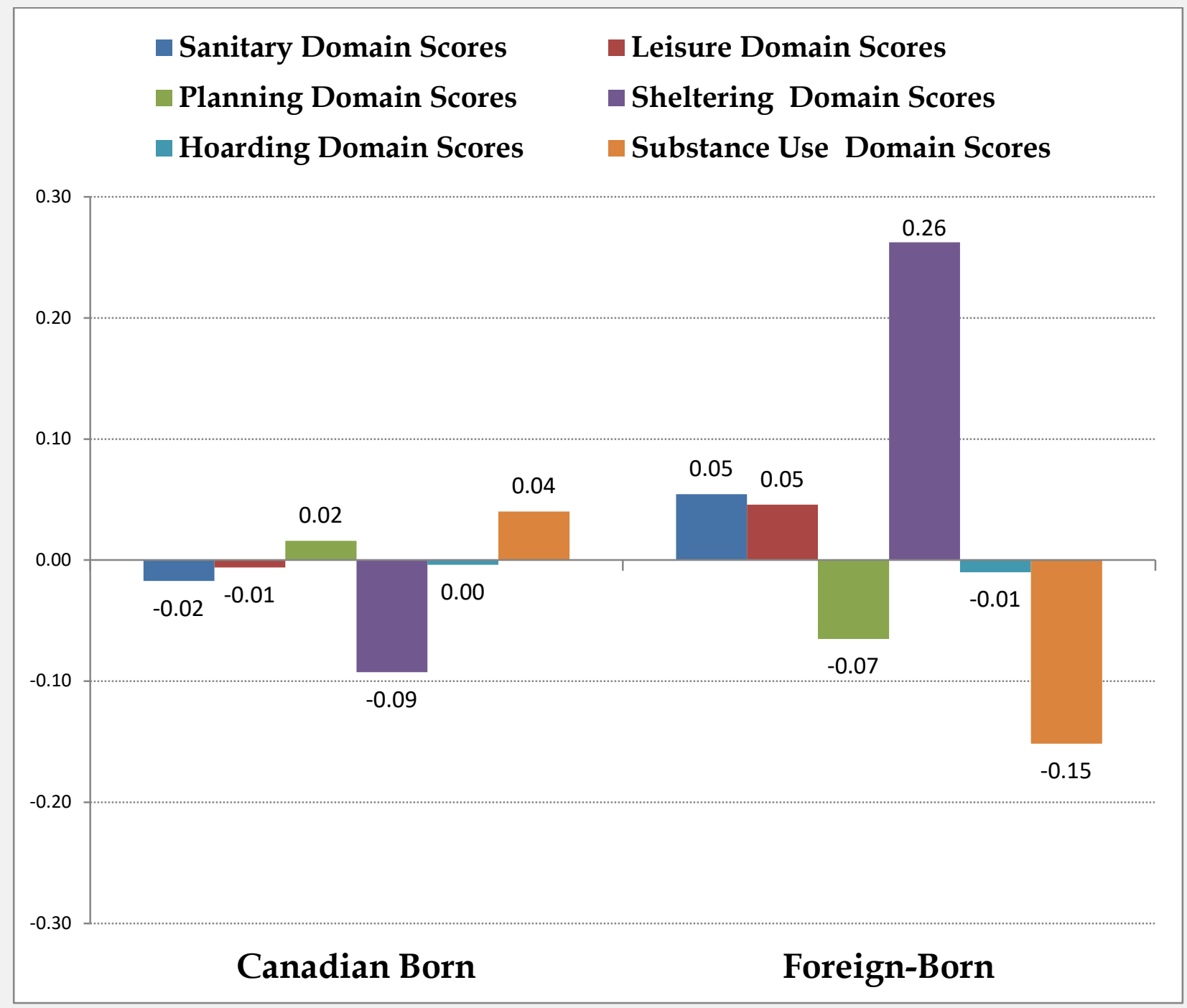

* $t$-tests for the Sheltering and Substance Use scores significant at the $\mathrm{p}<.05$ level. Source: Combined survey, CPSS Series $2 \& 4$ cycles 2020, Statistics Canada 
As final stage in the analysis and to identify the most consistent predictors of weekly activity domain scores, linear regressions were carried out having each domain score as dependent variable. These regression models accounted for modest proportions of the variance explained in the dependent variables and did not surpass 9\%. Controlling for other socio-demographic and psychosocial characteristics, immigrant status had negligible impacts on the Sanitary, Leisure, Planning and Hoarding scores (i.e. regression coefficients not statistically significant). However, controlling for other covariates and compared to the Canadian-born, being an immigrant increased the Sheltering scores by .09 standard deviation points and decreased the Substance Use scores by -.06 standard deviation points (both regression coefficients significant at the $\mathrm{p}<.05$ level). 
Chart 4: Average Component Scores of Sheltering and Substance Use Domains of Weekly Items*, Canadian-born and Foreign-born, Canada 2020

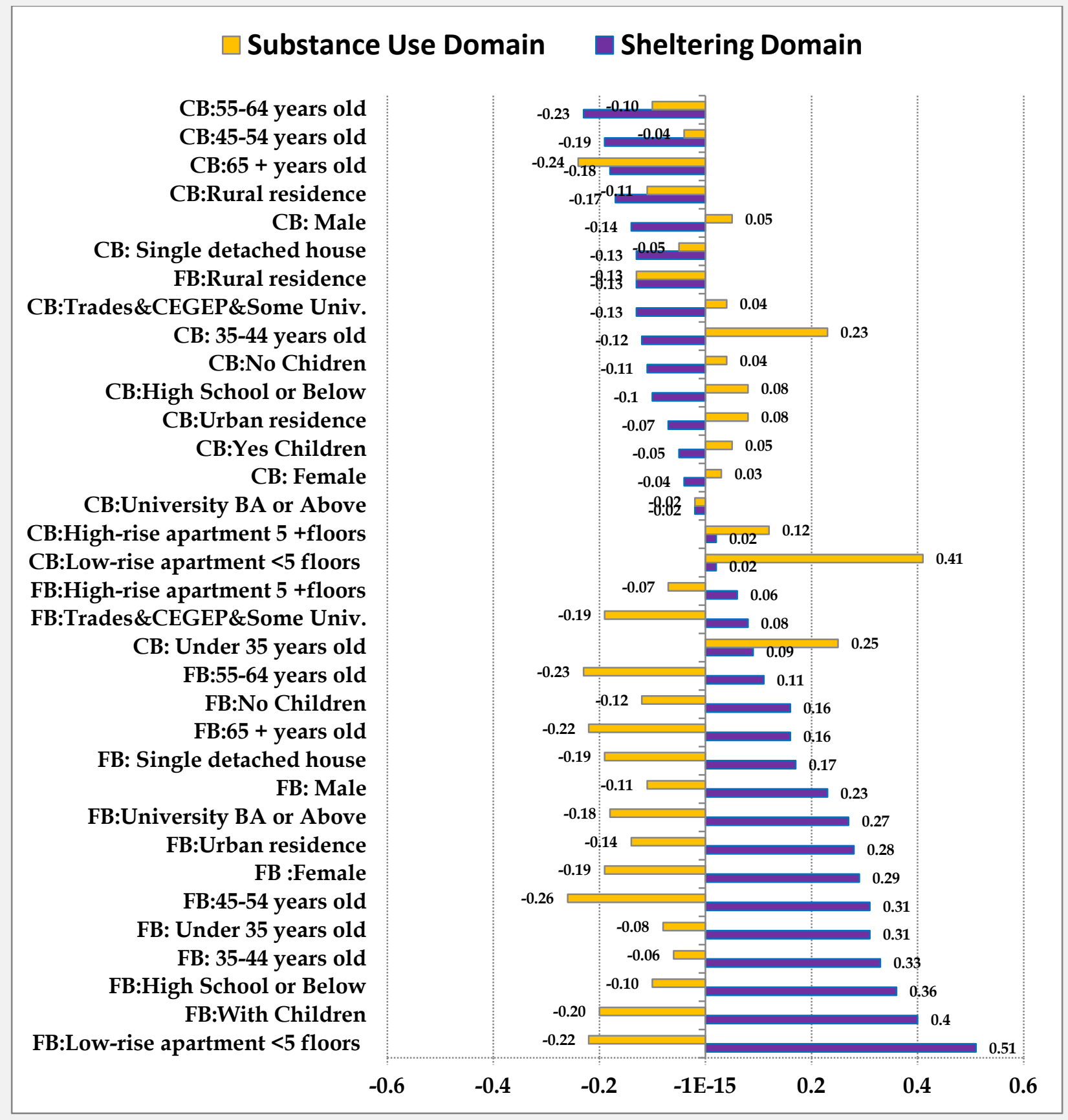

* Symbols: CB=Canadian-Born, FB=Foreign-Born. Source: Combined Survey, CPSS Series $2 \& 4$ cycles 2020, Statistics Canada

\subsection{Conclusions}

This study looked at typical weekly activities carried out by the Canadian-born and Foreign-born during the first wave of the COVID-19 pandemic in Canada. PCA became a valuable statistical tool to take a closer look at the behaviour of members in these two sub-populations. It should be noted that the available public versions of the CPSS surveys used for this study regrettably did not 
contain any details on the length of stay of those who were foreign-born, racial backgrounds, or provincial residences and provided only a broad snapshot on selected weekly activities. The voluntary nature of the surveys also raises the issue of potential biases due to the self-selection process. Despite these limitations, however, several interesting stories emerged from the analysis of the combined CPSS 2 and CPSS 4 data.

Firstly, this study did not find any substantial differences in the types of weekly activities performed by members of the Canadian-born and foreign-born subpopulations concerning following sanitary guidelines, communication activities, hoarding and leisure type of activities. Secondly, however, the Foreign-born were different from Canadian-born in terms of their sheltering and drug use type of activities. Immigrants "hunkered down" during the pandemic period. They canceled more their travel plans and maintained physical and mental health through indoor exercises (rather than outdoor exercise) and spiritual activities such as meditation. Also, they become more experienced in making better food choices (i.e. what to buy and eat). These behaviours suggest that the household immigrant "bubble"12 may have been at the center of their sheltering-related activities. This divergence may be partly explained by their prevalent types of residential accommodations such as living in apartment buildings and other more crowded housing arrangements but this remains to be explored in more detail ${ }^{13}$. Thirdly, focusing on the findings pertaining to substance use, it was evident that immigrants did not use alcohol, smoking and/or cannabis use as means to manage pandemic stress as much as the Canadian-born. In this sense, they display "healthier" habits which, however, have been found to deteriorate with longer periods of stay in Canada (see De Maio and Kemp,2010).

More research is needed on how immigrants and non-immigrants carry on with their daily lives during the stressful pandemic period. Qualitative studies may play a larger role in this regard. This may be particularly pressing in light of findings that many recent immigrants (who are most visible minorities) may be "essential "workers who live in more crowded physical environments where social distancing is difficult. The pandemic is creating a series of "vulnerable" groups in the labour market (Amarasingue et. al., 2020). Further avenues of investigation include a focus on the weekly activities of those who are performing the riskiest jobs in terms of disease exposure and proximity to other people.

\subsection{References}

Alfaroy,L; Faiaz,E.; Lamersdorfx, N.; and, Saidi,F (2020) Social Interaction in Pandemics: Fear, Altruism, and Reciprocity (2020), NBER Working Paper Series, No. 27134.

Amarasinghe, U.; Motha-Pollock, A.; Felder, M.; and Oschinski, M. (2020). COVID-19 and Ontario's Sales and Service Workers: Who is most vulnerable?, Mars Report, retrieved from:

https:// www.marsdd.com/research-and-insights/covid-19-and-ontarios-sales-and-service-workerswho-is-most-vulnerable/

Cockerham, W. 2005. Health Weekly Theory and the Convergence of Agency and Structure, Journal of Health and Social Behavior 2005, Vol 46 (March): 51-67.

Da Silva, M.; Barbosa-Rocha, R.; Buheji, M.; Jahrami, H.; and Costa-Cuhna, K. (2020). A Systematic Review of the Prevalence of Anxiety Symptoms During Coronavirus Epidemics, Journal of Health Psychology 1 (11).

\footnotetext{
12 A "bubble" is an unofficial term used in Canada to describe people with whom the individual feels comfortable spending time during the pandemic.

${ }^{13}$ Immigrants had a higher proportions of households with 4+ members compared to the Canadian-born: $20 \%$ to $13 \%$.
} 
De Maio, F.G and Kemp, B. (2010) The Deterioration of Health status Among Immigrants to Canada, Global Public Health, 5:5, 462-478.

Everitt, B.S. and Dunn, G. (2001). Applied Multivariate Analysis. London: Hodder Arnold.

Greenacre, M. Biplots in Practice. (2010). Fundacion BBVA Publication, Bilbao, Spain.

Hume, J. (2000). The Forgotten 1918 Influenza Epidemic and Press Portrayal of Public Anxiety, Journalism and Mass Communication Quarterly, Vol 77 (4), pp.898-915.

Hunt, A. (1999). Anxiety and Social Explanation: Some Anxieties about Anxiety, Journal of Social History, Vol. 32 (3),

IRPP. (2020). "Canadas-covid-19-blind-spots-on-race-immigration-and-labour" https:// policyoptiono.irpp.org/magazines/may-2020/canadas-covid-19-blind-spots-on-raceimmigration-and-labour/retrieved April 17,2021

Jobson, J.D. (1992). Applied Multivariate Data Analysis, Vol II : Categorical and Multivariate Methods New York: Springer-Verlag.

Kelly, J.R. (2019). Leisure Activities and Interaction, London: Routledge.

Lazarus, R. S., \& Folkman, S. (1984). Stress, Appraisal, and Coping. New York: Springer.

Maeng, L. Y. , \& Milad, M. R. (2015). Sex differences in anxiety disorders: Interactions between fear, stress, and gonadal hormones. Hormones and Behavior , $76,106-117$.

https://doi.org/10.1016/j.yhbeh.2015.04.002

Manderson, L., and Levine, S. (2020) COVID-19, Risk, Fear, and Fall-out, Lenore Manderson \& Susan Levine (2020) COVID-19, Risk, Fear, and Fall-out, Medical Anthropology, 39:5, 367-370, DOI:

$10.1080 / 01459740.2020 .1746301$

OECD (2020). "Policy Responses to Coronavirus (COVID-19) What is the impact of the COVID-19 pandemic on immigrants and their children?", https:// www.oecd.org/coronavirus/policyresponoes/ what-is-the-impact-of-the-covid-19-pandemic-on-immigrants-and-their-children-e7cbb7de/ Retrieved April 17,2021

Strong, P. (1990) Epidemic Psychology: A Model. Sociology of Health and Illness, Vol. 12(3), pp.249-59.

World Health Organization-WHO. (2020). Mental Health and Psychosocial Considerations During COVID-19; Outreach; World Health Organization: Genève, Switzerland, 2020. 